\title{
Minimum System Sensitivity Study of Linear Discrete Time Systems for Fault Detection
}

\author{
Xiaobo Li and Hugh H. T. Liu \\ Institute for Aerospace Studies, University of Toronto, Toronto, ON, Canada M3H 5T6 \\ Correspondence should be addressed to Hugh H. T. Liu; liu@utias.utoronto.ca
}

Received 2 September 2012; Accepted 15 January 2013

Academic Editor: Bin Jiang

Copyright ( $92013 \mathrm{X}$. Li and H. H. T. Liu. This is an open access article distributed under the Creative Commons Attribution License, which permits unrestricted use, distribution, and reproduction in any medium, provided the original work is properly cited.

\begin{abstract}
Fault detection is a critical step in the fault diagnosis of modern complex systems. An important notion in fault detection is the smallest gain of system sensitivity, denoted as $\mathscr{H}_{-}$index, which measures the worst fault sensitivity. This paper is concerned with characterizing $\mathscr{H}_{-}$index for linear discrete time systems. First, a necessary and sufficient condition on the lower bound of $\mathscr{H}_{-}$index in finite time horizon for linear discrete time-varying systems is developed. It is characterized in terms of the existence of solution to a backward difference Riccati equation with an inequality constraint. The result is further extended to systems with unknown initial condition based on a modified $\mathscr{H}_{-}$index. In addition, for linear time-invariant systems in infinite time horizon, based on the definition of the $\mathscr{H}_{-}$index in frequency domain, a condition in terms of algebraic Riccati equation is developed. In comparison with the well-known bounded real lemma, it is found that $\mathscr{H}_{-}$index is not completely dual to $\mathscr{H}_{\infty}$ norm. Finally, several numerical examples are given to illustrate the main results.
\end{abstract}

\section{Introduction}

Fault diagnosis is an important function in modern systems development and operation. It aims at detecting and identifying failure as soon as it occurs so as to avoid severe performance deterioration. Model-based fault diagnosis approach has attracted a great deal of interest in the past several decades (see [1-4] and the references therein). As part of the fault diagnosis process, the specific aim of fault detection is generally acknowledged to design a filter that generates residual signal to predict the occurrence of faults [1]. The fault detection filter design for linear time-invariant systems has been widely investigated $[1,5]$. The optimization-based fault detection designs have been widely investigated [611]. Furthermore, there are some studies aiming at making the tradeoff of two objectives: robustness to disturbances and sensitivity to faults. Based on this tradeoff, many design criteria and the corresponding techniques have been proposed [12-19]. In these works, the smallest sensitivity of system output to input, termed $\mathscr{H}_{-}$index, is widely used for measuring the smallest sensitivity of residual to faults in frequency domain $[5,13,15,16,19,20]$. In other words, it is used to represent the worst fault sensitivity $[1,13,15,16,21$ 23]. To characterize this index, we will provide new approach for fault detection design. In [16], Liu et al. extended the definition of $\mathscr{H}_{-}$index as the smallest singular value over all frequency range and derived the condition to characterize it in terms of linear matrix inequality (LMI) and algebraic Riccati equation.

Although much work on fault detection based on the $\mathscr{H}_{\text {_ }}$ index in frequency domain is available, very few work was reported in public literature that addresses $\mathscr{H}_{-}$index in time domain. In [21], Li and Zhou derived a fault residual generator with maximizing the fault sensitivity in terms of $\mathscr{H}_{\text {_ }}$ index in finite time horizon under the disturbance sensitivity constraint. The result is further extended to discretetime systems in [24, 25]. However, no explicit condition to characterize $\mathscr{H}_{-}$index was given in [21]. In our recent work $[26,27]$, we developed conditions to characterize the $\mathscr{H}_{-}$ index of linear time-varying systems in finite time horizon.

Many industrial systems are discrete-time systems. They exhibit different properties from continuous-time systems and are also subject to various failures. To investigate the fault detection of discrete-time systems such as the $\mathscr{H}_{-}$index thus 
has considerable merit. It will not only provide guidance and design criterion for fault detection of discrete-time systems, but also inspire the new approach for fault detection design. As such, this paper aims at characterizing the $\mathscr{H}_{-}$index of the linear discrete time systems. At first, from perspective of time domain, we show that the lower bound of the $\mathscr{H}_{-}$index in finite time horizon for linear discrete time-varying systems can be characterized as existence of solution for a certain backward difference Riccati equation with an inequality constraint. The result is further extended to systems with unknown initial condition based on a modified $\mathscr{H}_{-}$index to emphasize the effect of initial state. On the other hand, in order to characterize $\mathscr{H}_{-}$index from frequency domain, we also develop the necessary and sufficient condition for $\mathscr{H}_{\text {_ }}$ index of linear discrete time-invariant systems, which is given in terms of algebraic Riccati equation. An equivalent LMI condition is also derived. Surprisingly, by comparing this result with the famous bounded real lemma [28-30], we find that $\mathscr{H}_{-}$index is not completely dual to $\mathscr{H}_{\infty}$ norm.

The remaining part of this paper is organized as follows. Some relevant notations and definitions are given in Section 2. In Section 3, we develop a necessary and sufficient condition to characterize the $\mathscr{H}_{-}$index of linear discrete time-varying systems in finite time horizon. The result is further extended to systems with nonzero initial condition in Section 4. In Section 5, we investigate $\mathscr{H}_{-}$index from frequency domain for linear discrete time-invariant systems. The comparison with the famous bounded real lemma is given in Section 6. To illustrate our results and demonstrate its application in fault detection field, several examples are provided in Section 7. Section 8 is our conclusion.

\section{Notations and Definitions}

The notation adopted in this paper is fairly standard. The set of $m \times n$ real (complex) matrices is denoted as $\mathbb{R}^{m \times n}$ $\left(\mathbb{C}^{m \times n}\right)$. The set of $m \times 1$ real (complex) vectors is denoted as $\mathbb{R}^{m}\left(\mathbb{C}^{m}\right)$. For a matrix $A \in \mathbb{R}^{m \times n}$, we use $A^{\prime}$ to denote its transpose. For a matrix $A \in \mathbb{C}^{m \times n}$, we use $A^{*}$ to denote its complex conjugate transpose. For a matrix $A \in \mathbb{C}^{m \times m}$, $\lambda(A)$ represents all the eigenvalues of $A$, and $A^{-1}$ is for its inverse if it exists. A matrix $A \in \mathbb{R}^{m \times n}$ is stable if all the eigenvalues are strictly in the unit circle. For a symmetric matrix $A=A^{*} \in \mathbb{C}^{n \times n}, \bar{\lambda}(A)$ represents the largest eigenvalue of $A$ and $\underline{\lambda}(A)$ represents the smallest eigenvalue of $A$. For $A \in \mathbb{C}^{m \times n}, \bar{\sigma}(A)=\sqrt{\bar{\lambda}\left(A A^{*}\right)}=\sqrt{\bar{\lambda}\left(A^{*} A\right)}$ denotes the largest singular value of $A$ and $\underline{\sigma}(A)=\sqrt{\lambda\left(A^{*} A\right)}$ denotes the smallest singular value of $A \overline{\text { if }}$ it is not wide. The $n \times n$ identity matrix is denoted as $I_{n}$, with the subscript dropped if they can be inferred from context. $A>0(\geq 0,<0, \leq$ 0 ) means that $A$ is positive definite (positive semidefinite, negative definite, and negative semidefinite). We denote by $A^{1 / 2}$ the square root of a positive semidefinite matrix $A$ if $A^{1 / 2}=\left(A^{1 / 2}\right)^{*}>0$ and $A=A^{1 / 2} A^{1 / 2}$. For a positive integer $T>0$, let $\mathscr{L}_{2}[0, T]$ denote the usual Hilbert space of square summable sequences endowed with usual inner product and norm (denoted as $\langle\cdot, \cdot\rangle_{2,[0, T]}$ and $\|\cdot\|_{2,[0, T]}$, resp.). Specifically, for a sequence $x(t)$, its 2-norm in finite time horizon is given by $\|x\|_{2,[0, T]}=\sqrt{\sum_{0}^{T} x^{\prime}(t) x(t) d t}$. Throughout the paper, we compress the notation and write $\mathscr{L}_{2}[0, \infty)$ or $\mathscr{L}_{2}[0, T]$ as $\mathscr{L}_{2}$ and $\|\cdot\|_{2,[0, T]}$ as $\|\cdot\|_{2}$ whenever there is no confusion.

Consider the following linear discrete time-varying system $\mathbf{G}$ with zero initial condition:

$$
\begin{gathered}
x(t+1)=A(t) x(t)+B(t) w(t), \quad x(0)=0, \\
y(t)=C(t) x(t)+D(t) w(t),
\end{gathered}
$$

where $x \in \mathbb{R}^{n}, w \in \mathbb{R}^{p}$, and $y \in \mathbb{R}^{q}$ are the states, system input, and system output, respectively, and $A(t), B(t), C(t)$, and $D(t)$ are real time-varying coefficients with compatible dimensions. $t=\ldots,-1,0,1, \ldots$ represents time sequence. Time $t$ is omitted sometimes for simplicity. The initial time can be any integer $t_{0}$. In this paper, $t_{0}=0$ is assumed without loss of generality. We call that $\mathbf{G}$ is a tall (wide, or square) system if $p<q(p>q$, or $p=q)$.

The minimal system sensitivity is very widely used in fault detection field to measure the worst fault sensitivity $[5,13,15,16,19,20]$. In frequency domain, it is defined as the minimal singular values over the whole frequency range. However, this definition makes no sense for the time-varying systems. Therefore, we consider the following definition.

Definition 1 (see [24]). $\mathscr{H}_{-}$index for system (1) in finite time horizon $[0, T]$ ( $T$ is a positive integer) is defined as

$$
\begin{aligned}
\|\mathbf{G}\|_{-}^{[0, T]} & :=\inf _{w \neq 0} \frac{\|\mathbf{G} w\|_{2,[0, T]}}{\|w\|_{2,[0, T]}}=\inf _{w \neq 0} \frac{\|y\|_{2,[0, T]}}{\|w\|_{2,[0, T]}} \\
& =\inf _{w \neq 0} \sqrt{\frac{\sum_{t=0}^{T} y^{\prime}(t) y(t)}{\sum_{t=0}^{T} w^{\prime}(t) w(t)}},
\end{aligned}
$$$$
\forall w \in \mathscr{L}_{2}[0, T], w \neq 0 .
$$

Remark 2. This definition characterizes the smallest sensitivity of system $\mathbf{G}$ from input $w$ to output $y$ in time domain. It can be used in fault detection field to measure the minimal fault sensitivity of fault signal [1]. Specifically, by assuming that system $\mathbf{G}$ is the system from fault signal $w$ to residual $y$, then $\|\mathbf{G}\|_{-}^{[0, T]}$ measures the minimal fault sensitivity. This definition is dual to $\mathscr{H}_{\infty}$ norm for the largest system sensitivity [30].

In this paper, we assume that system $\mathbf{G}$ is tall or square $(p \leq q)$, since the $\mathscr{H}_{-}$index of wide systems is always zero; that is, $\|\mathbf{G}\|_{-}=0$. In contrast, $\mathscr{H}_{\infty}$ norm is applicable to wide systems. The goal of this paper is to characterize this index, so that it can be easily used in the fault detection field.

\section{Characterizing $\mathscr{H}_{-}$Index of Linear Discrete Time-Varying Systems in Finite Time Horizon}

In this section, we develop a condition to characterize the lower bound of $\mathscr{H}_{-}$index of linear discrete time-varying systems in finite time horizon, stated in the following theorem. 
Theorem 3. Consider the linear discrete time-varying system G. Let $\gamma$ be a nonnegative scalar; that is, $\gamma \geq 0$. The following two conditions are equivalent:

(1) $\|\mathbf{G}\|_{-}^{[0, T]}>\gamma$,

(2) there exist $\{P(t)\}_{t=0}^{T}$ to difference the Riccati equation

$$
\begin{aligned}
P(t-1) & \\
= & A^{\prime}(t) P(t) A(t)+C^{\prime}(t) C(t) \\
& +\left[D^{\prime}(t) C(t)+B^{\prime}(t) P(t) A(t)\right]^{\prime} R^{-1}(t) \\
& \times\left[D^{\prime}(t) C(t)+B^{\prime}(t) P(t) A(t)\right]
\end{aligned}
$$

with $P(T)=0$, where $R(t)=\gamma^{2} I-D^{\prime}(t) D(t)-$ $B^{\prime}(t) P(t) B(t)<0$.

Remark 4. It can be seen that the lower bound of $\mathscr{H}_{-}$index of linear discrete time-varying system $\mathbf{G}$ can be characterized as a difference Riccati equation (3) and inequality constraint $R<0$. In other words, verification of Item 2 can be used to evaluate the $\mathscr{H}_{-}$index of a linear discrete time-varying system. It can be used in fault detection to measure the worst fault sensitivity of a system under a fault detection filter.

\subsection{Proof of (2) $\Rightarrow(1)$}

Proof. Note that $P(T)=0$ and $x(0)=0$. It follows that

$$
\begin{aligned}
& \sum_{t=0}^{T}\left[x^{\prime}(t+1) P(t) x(t+1)-x^{\prime}(t) P(t-1) x(t)\right] \\
& =x^{\prime}(T+1) P(T) x(T+1)-x^{\prime}(0) P(-1) x(0)=0 .
\end{aligned}
$$

We have the following parameterized performance index for the linear discrete time-varying system (1):

$$
\begin{aligned}
& J(\gamma):=\sum_{t=0}^{T}\left(y^{\prime} y-\gamma^{2} w^{\prime} w\right) \\
&= \sum_{t=0}^{T}\left\{(C x+D w)^{\prime}(C x+D w)-\gamma^{2} w^{\prime} w\right\} \\
&= \sum_{t=0}^{T}\left\{\left(x^{\prime} C^{\prime} C x+w^{\prime} D^{\prime} C x+x^{\prime} C^{\prime} D w+w^{\prime} D^{\prime} D w\right)\right. \\
&\left.-\gamma^{2} w^{\prime} w\right\}
\end{aligned}
$$

$$
\begin{aligned}
& +\sum_{t=0}^{T}\left\{x^{\prime}(t+1) P(t) x(t+1)-x^{\prime}(t) P(t-1) x(t)\right\} \\
& -x^{\prime}(T+1) P(T) x(T+1)+x^{\prime}(0) P(-1) x(0) \\
& =\sum_{t=0}^{T}\left\{\left(x^{\prime} C^{\prime} C x+w^{\prime} D^{\prime} C x+x^{\prime} C^{\prime} D w+w^{\prime} D^{\prime} D w\right)\right. \\
& \left.\quad-\gamma^{2} w^{\prime} w\right\} \\
& +\sum_{t=0}^{T}\left\{(A x+B w)^{\prime} P(t)(A x+B w)\right. \\
& \left.\quad-x^{\prime}(t) P(t-1) x(t)\right\} \\
& =x_{t=0}^{T}(T+1) P(T) x(T+1)+x^{\prime}(0) P(-1) x(0) \\
& +x^{\prime}\left(A^{\prime} P A+C^{\prime} C-P(t-1)\right) x \\
& \left.+w^{\prime}\left(D^{\prime} D-\gamma^{2} I+B^{\prime} P B\right) w\right\} .
\end{aligned}
$$

To substitute the Riccati equation (3) in the expression of $J(\gamma)$, we have

$$
\begin{aligned}
& J(\gamma)=\sum_{t=0}^{T}\left\{-x^{\prime}\left(D^{\prime} C+B^{\prime} P A\right)^{\prime} R^{-1}\left(D^{\prime} C+B^{\prime} P A\right) x\right. \\
& +x^{\prime}\left(C^{\prime} D+A^{\prime} P B\right) w+w^{\prime}\left(D^{\prime} C+B^{\prime} P A\right) x \\
& \left.+w^{\prime}\left(D^{\prime} D-\gamma^{2} I+B^{\prime} P B\right) w\right\} \\
& =\sum_{t=0}^{T}\left\{-x^{\prime}\left(D^{\prime} C+B^{\prime} P A\right)^{\prime} R^{-1}\left(D^{\prime} C+B^{\prime} P A\right) x\right. \\
& +x^{\prime}\left(C^{\prime} D+A^{\prime} P B\right) w+w^{\prime}\left(D^{\prime} C+B^{\prime} P A\right) x \\
& \left.-w^{\prime} R w\right\} \\
& =\sum_{t=0}^{T}\left[w-R^{-1}\left(D^{\prime} C+B^{\prime} P A\right) x\right](-R) \\
& \times\left[w-R^{-1}\left(D^{\prime} C+B^{\prime} P A\right) x\right] .
\end{aligned}
$$

Define $\widetilde{w}:=R^{-1}\left(D^{\prime} C+B^{\prime} P A\right) x$. We then have

$$
J(\gamma)=\sum_{t=0}^{T}(w-\widetilde{w})^{\prime}(-R)(w-\widetilde{w}) .
$$

Let $\mathbf{L}$ be the operator from $w$ to $w-\widetilde{w}$, written as

$$
\begin{gathered}
x(t+1)=A x+B w \\
w-\widetilde{w}=-R^{-1}\left(D^{\prime} C+B^{\prime} P A\right) x+w .
\end{gathered}
$$


Its inverse operator $\mathrm{L}^{-1}$ exists and is given by

$$
\begin{gathered}
x(t+1)=\left[A+B R^{-1}\left(D^{\prime} C+B^{\prime} P A\right)\right] x+B(w-\widetilde{w}), \\
w=R^{-1}\left(D^{\prime} C+B^{\prime} P A\right) x+(w-\widetilde{w}) .
\end{gathered}
$$

It follows that

$$
\begin{aligned}
J(\gamma) & =\sum_{t=0}^{T}(w-\widetilde{w})^{\prime}(-R)(w-\widetilde{w}) \\
& =\left\|(-R)^{1 / 2} \mathbf{L}(w)\right\|_{2}^{2} \geq \epsilon\|w\|_{2}^{2}>0
\end{aligned}
$$

for some positive number $\epsilon$ and $w \neq 0$.

\subsection{Proof of $(1) \Rightarrow(2)$}

Proof. Assume $\|\mathbf{G}\|_{-}^{[0, T]}>\gamma$. Then $J(\gamma)>0$. Choose $P(T)=0$. Then choose $\{P(t)\}_{t=0}^{T-1}$ as that in the Riccati equation (3). Thus, the existence of solution $\{P(t)\}_{t=0}^{T-1}$ depends on the existence of $\{R(t)\}_{t=1}^{T}$ from the Riccati equation (3). In fact, the feasibility of inverse of $R$ is implied by $J(\gamma)>0$. At first, it can be verified that

$$
\begin{aligned}
& J(\gamma) \\
& =\sum_{t=0}^{T}\left[\begin{array}{c}
x \\
w
\end{array}\right]^{\prime}\left[\begin{array}{cc}
A^{\prime} P A+C^{\prime} C-P(t-1) & A^{\prime} P B+C^{\prime} D \\
B^{\prime} P A+D^{\prime} C & -R
\end{array}\right] \\
& \times\left[\begin{array}{l}
x \\
w
\end{array}\right] .
\end{aligned}
$$

Thus, $J(\gamma)>0$ for any nonzero $w$ implies that $R(t)<0$ for $t=0,1, \ldots, T$ (otherwise, we can choose $P(t-1)$ to make $J(\gamma)$ nonpositive). Furthermore, it implies that $R$ is invertible.

Corollary 5. If $\mathbf{G}$ is square $(p=q)$, an alternative condition for condition (2) is that there exist $\{P(t)\}_{t=0}^{T}$ to forward difference Riccati equation:

$$
\begin{aligned}
P(t+1)= & A(t) P(t) A^{\prime}(t)+B(t) B^{\prime}(t) \\
& +\left[D B^{\prime}+C P(t) A^{\prime}\right]^{\prime} R^{-1}(t)\left[D B^{\prime}+C P(t) A^{\prime}\right],
\end{aligned}
$$

with $P(0)=0$, where $R(t)=\gamma^{2} I-D(t) D^{\prime}(t)-C(t) P(t) C^{\prime}(t)<$ 0 .

Proof. The proof is based on the adjoint system $\mathbf{G}^{\sim}$ :

$$
\begin{gathered}
p(t-1)=A^{\prime}(t) p(t)+C^{\prime}(t) y(t), \quad p(T)=0, \\
w(t)=B^{\prime}(t) p(t)+D^{\prime}(t) y(t) .
\end{gathered}
$$

Now we show that $\|\mathbf{G}\|_{-}=\left\|\mathbf{G}^{\sim}\right\|_{-}$if $\mathbf{G}$ is square. For any $y \in$ $\mathscr{L}_{2}$

$$
\begin{aligned}
\left\|\mathbf{G}^{\sim} y\right\|_{2}^{2} & =\left\langle\mathbf{G}^{\sim} y, \mathbf{G}^{\sim} y\right\rangle=\left\langle\mathbf{G G}^{\sim} y, y\right\rangle \\
& =\left\|\mathbf{G G}^{\sim} y\right\|_{2}\|y\|_{2} \geq\left\|\mathbf{G}_{-}\right\| \mathbf{G}^{\sim} y\left\|_{2}\right\| y \|_{2},
\end{aligned}
$$

which implies that

$$
\left\|\mathbf{G}^{\sim} y\right\|_{2} \geq\|\mathbf{G}\|_{-}\|y\|_{2}
$$

when $\mathbf{G}$ is not a tall matrix (note that $\left\|\mathbf{G}^{\sim} y\right\|_{2}=0$ for some $y$ if $\mathbf{G}$ is tall). Thus, $\left\|\mathbf{G}^{\sim}\right\|_{-} \geq\|\mathbf{G}\|_{-}$if $\mathbf{G}$ is wide or square. On the other hand, since $\left(\mathbf{G}^{\sim}\right)^{\sim}=\mathbf{G}$, we also have $\left\|\mathbf{G}^{\sim}\right\|_{-} \leq\|\mathbf{G}\|_{-}$ if $\mathbf{G}$ is tall or square. Hence, $\left\|\mathbf{G}^{\sim}\right\|_{-}=\|\mathbf{G}\|_{-}$if $\mathbf{G}$ is square. Therefore, we only need to characterize the condition for adjoint system $\mathbf{G}^{\sim}$. The rest follows Theorem 3 .

\section{Extension to Unknown Initial Condition}

In Section 3, we developed a necessary and sufficient condition to characterize $\mathscr{H}_{-}$index for linear discrete timevarying system with zero initial condition. However, the initial condition may also make significant effect on the system dynamics and characteristics. From the perspective of fault detection, it could bring unignored effect to the worst fault sensitivity. Thus, it is more reasonable to consider a modified $\mathscr{H}_{-}$index with considering the initial condition.

In this section, the effect of unknown initial condition as well as the current state is taken into consideration by employing a modified $\mathscr{H}_{-}$index. It will be shown that the $\mathscr{H}_{\text {_ }}$ index with unknown initial condition is characterized as a backward difference Riccati equation with an inequality condition.

Consider the following linear discrete time-varying system $\mathbf{G}$ with unknown initial condition:

$$
\begin{gathered}
x(t+1)=A(t) x(t)+B(t) w(t), \quad x(0)=x_{0}, \\
y(t)=C(t) x(t)+D(t) w(t),
\end{gathered}
$$

where $x \in \mathbb{R}^{n}, w \in \mathbb{R}^{p}$, and $y \in \mathbb{R}^{q}$ are the states, system input, and system output, respectively, and $A(t), B(t), C(t)$, and $D(t)$ are real time-varying coefficients with compatible dimensions. Sometimes time $t$ is omitted for simplicity. The initial time can be any integer $t_{0}$. Here we assume $t_{0}=0$ without loss of generality. The initial state $x_{0}$ is assumed to be unknown.

Definition 6. The modified $\mathscr{H}_{-}$index in finite time horizon $[0, T]$ ( $T$ is a positive integer) for linear discrete time-varying system (16) is defined as

$$
\begin{array}{r}
\|\mathbf{G}\|_{-}^{[0, T]}:=\inf _{w, x_{0}} \sqrt{\frac{\sum_{t=0}^{T} y^{\prime}(t) y(t)+x^{\prime}(T+1) Q x(T+1)}{\sum_{t=0}^{T} w^{\prime}(t) w(t)+x^{\prime}(0) S x(0)},} \\
\forall w \in \mathscr{L}_{2}[0, T], x_{0} \in \mathbb{R}^{n},
\end{array}
$$$$
\text { s.t. } \quad \sum_{t=0}^{T} w^{\prime}(t) w(t)+x^{\prime}(0) S x(0) \neq 0 \text {. }
$$

Remark 7. In the modified $\mathscr{H}_{-}$index, $Q>0$ and $S>0$ are used to emphasize the effects of states at time instants $T+1$ and 0 , respectively. Different $Q$ and $S$ imply different emphases on the initial and final states. When $Q=0$, it turns out to be the definition in [24]. When both $Q$ and $S$ are zero, it turns out to be the standard definition (Definition 1). 
We also assume that $\mathbf{G}$ is tall or square, since it makes no sense to characterize $\mathscr{H}_{-}$index for wide systems.

The following theorem characterizes the modified $\mathscr{H}_{-}$ index in terms of a backward difference Riccati equation with an inequality condition.

Theorem 8. Consider linear discrete time-varying system (16). Let $\gamma$ be a nonnegative scalar; that is, $\gamma \geq 0$. The following two conditions are equivalent:

(1) $\|\mathbf{G}\|_{-}^{[0, T]}>\gamma$,

(2) there exist $\{P(t)\}_{t=0}^{T}$ such that $R(t)=\gamma^{2} I-D^{\prime} D-$ $B^{\prime} P(t) B<0$, and

$P(t-1)$

$$
\begin{aligned}
= & A^{\prime}(t) P(t) A(t)+C^{\prime}(t) C(t) \\
& +\left[D^{\prime}(t) C(t)+B^{\prime}(t) P(t) A(t)\right]^{\prime} R^{-1}(t) \\
& \times\left[D^{\prime}(t) C(t)+B^{\prime}(t) P(t) A(t)\right]
\end{aligned}
$$

with $P(T)=Q$ and $P(-1)>\gamma^{2} S$.

Remark 9. The difference from the zero initial condition case is that the terminal condition for $(18)$ is $P(T)=Q$ instead of 0 .

Corollary 10. Assume that $\mathbf{G}$ is square $(p=q)$. Then $\|\mathbf{G}\|_{-}^{[0, T]}>\gamma(\gamma \geq 0)$ if and only if there exist $\{P(t)\}_{t=0}^{T}$ to the following forward difference Riccati equation:

$$
\begin{aligned}
P(t+1)= & A P(t) A^{\prime}+B B^{\prime}+\left[D B^{\prime}+C P(t) A^{\prime}\right]^{\prime} R^{-1}(t) \\
& \times\left[D B^{\prime}+C P(t) A^{\prime}\right]
\end{aligned}
$$

with $P(0)=S$ and $P(T+1)>Q / \gamma$, where $R(t)=\gamma^{2} I-$ $D(t) D^{\prime}(t)-C(t) P(t) C^{\prime}(t)<0$.

\subsection{Proof of $(2) \Rightarrow(1)$}

Proof. Note that we have the relation

$$
\begin{aligned}
& \sum_{t=0}^{T}\left[x^{\prime}(t+1) P(t) x(t+1)-x^{\prime}(t) P(t-1) x(t)\right] \\
& =x^{\prime}(T+1) P(T) x(T+1)-x^{\prime}(0) P(-1) x(0) .
\end{aligned}
$$

We have the following parameterized performance index for discrete time-varying system (16):

$$
\begin{aligned}
J(\gamma)= & \sum_{t=0}^{T}\left(y^{\prime} y-\gamma^{2} w^{\prime} w\right)+x^{\prime}(T+1) Q x(T+1) \\
& -\gamma^{2} x^{\prime}(0) S x(0) \\
= & \sum_{t=0}^{T}\left\{(C x+D w)^{\prime}(C x+D w)-\gamma^{2} w^{\prime} w\right\} \\
& +x^{\prime}(T+1) Q x(T+1)-\gamma^{2} x^{\prime}(0) S x(0) \\
= & \sum_{t=0}^{T}\left\{\left(x^{\prime} C^{\prime} C x+w^{\prime} D^{\prime} C x+x^{\prime} C^{\prime} D w+w^{\prime} D^{\prime} D w\right)\right. \\
& +x^{\prime}(0)\left(P(-1)-\gamma^{2} S\right) x(0) . \\
& +x^{\prime}(T+1) Q x(T+1)-\gamma^{2} x^{\prime}(0) S x(0) \\
& +\sum_{t=0}^{T}\left\{x^{\prime}(t+1) P(t) x(t+1)-x^{\prime}(t) P(t-1) x(t)\right\} \\
& +x^{\prime}(T+1) P(T) x(T+1)+x^{\prime}(0) P(-1) x(0) \\
& +\sum_{t=0}^{T}\left\{(A x+B w)^{\prime} P(t)(A x+B w)-x^{\prime} P(t-1) x\right\} \\
= & \sum_{t=0}^{T}\left\{\left(x^{\prime} C^{\prime} C x+w^{\prime} D^{\prime} C x+x^{\prime} C^{\prime} D w+w^{\prime} D^{\prime} D w\right)\right. \\
& +x^{\prime}\left(T+\gamma^{2} w^{\prime} w\right\} \\
& \\
& \left.+\gamma^{\prime} D+A^{\prime} I\right) Q x(T+1)-\gamma^{2} x^{\prime}(0) S x(0)
\end{aligned}
$$

Note that $Q=P(T)$. To substitute the Riccati equation (18) in the expression of $J(\gamma)$, we have

$$
\begin{aligned}
J(\gamma)=\sum_{t=0}^{T}\{ & -x^{\prime}\left(D^{\prime} C+B^{\prime} P A\right)^{\prime} R^{-1}\left(D^{\prime} C+B^{\prime} P A\right) x \\
& +x^{\prime}\left(C^{\prime} D+A^{\prime} P B\right) w+w^{\prime}\left(D^{\prime} C+B^{\prime} P A\right) x \\
& \left.+w^{\prime}\left(D^{\prime} D-\gamma^{2} I+B^{\prime} P B\right) w\right\} \\
& +x^{\prime}(0)\left(P(-1)-\gamma^{2} S\right) x(0)
\end{aligned}
$$




$$
\begin{aligned}
& =\sum_{t=0}^{T}\left\{-x^{\prime}\left(D^{\prime} C+B^{\prime} P A\right)^{\prime} R^{-1}\left(D^{\prime} C+B^{\prime} P A\right) x\right. \\
& \quad+x^{\prime}\left(C^{\prime} D+A^{\prime} P B\right) w+w^{\prime}\left(D^{\prime} C+B^{\prime} P A\right) x \\
& \left.\quad \quad-w^{\prime} R w\right\} \\
& +x^{\prime}(0)\left(P(-1)-\gamma^{2} S\right) x(0) \\
& =\sum_{t=0}^{T}\left[w-R^{-1}\left(D^{\prime} C+B^{\prime} P A\right) x\right]^{\prime}(-R) \\
& \quad \times\left[w-R^{-1}\left(D^{\prime} C+B^{\prime} P A\right) x\right] \\
& +x^{\prime}(0)\left(P(-1)-\gamma^{2} S\right) x(0) .
\end{aligned}
$$

Define $\widetilde{w}:=R^{-1}\left(D^{\prime} C+B^{\prime} P A\right) x$. We then have

$$
\begin{aligned}
J(\gamma)= & \sum_{t=0}^{T}(w-\widetilde{w})^{\prime}(-R)(w-\widetilde{w}) \\
& +x^{\prime}(0)\left(P(-1)-\gamma^{2} S\right) x(0) .
\end{aligned}
$$

Let $\mathbf{L}$ be the operator from $w$ to $w-\widetilde{w}$, written as

$$
\begin{gathered}
x(t+1)=A x+B w, \\
w-\widetilde{w}=-R^{-1}\left(D^{\prime} C+B^{\prime} P A\right) x+w .
\end{gathered}
$$

Its inverse operator $\mathbf{L}^{-1}$ exists and is given by

$$
\begin{gathered}
x(t+1)=\left[A+B R^{-1}\left(D^{\prime} C+B^{\prime} P A\right)\right] x+B(w-\widetilde{w}), \\
w=R^{-1}\left(D^{\prime} C+B^{\prime} P A\right) x+(w-\widetilde{w}) .
\end{gathered}
$$

It follows that

$$
\begin{aligned}
J(\gamma)= & \sum_{t=0}^{T}(w-\widetilde{w})^{\prime}(-R)(w-\widetilde{w}) \\
& +x^{\prime}(0)\left(P(-1)-\gamma^{2} S\right) x(0) \\
= & \left\|(-R)^{1 / 2} \mathbf{L}(w)\right\|_{2}^{2}+x^{\prime}(0)\left(P(-1)-\gamma^{2} S\right) x(0) \\
\geq & \epsilon\|w\|_{2}^{2}+x^{\prime}(0)\left(P(-1)-\gamma^{2} S\right) x(0)>0
\end{aligned}
$$

for some positive number $\epsilon$ and $w \neq 0$ by noting the fact $P(-1)>\gamma^{2} S$. Obviously, it implies that $\|\mathbf{G}\|_{-}^{[0, T]}>\gamma$.

$$
\text { 4.2. } \operatorname{Proof} \text { of }(1) \Rightarrow(2)
$$

Proof. Assume $\|\mathbf{G}\|_{-}^{[0, T]}>\gamma$. Then $J(\gamma)>0$. Choose $X(T)=$ $Q$. Then choose $\{P(t)\}_{t=0}^{T-1}$ as that in the Riccati equation (18). It can be seen that the existence of $\{P(t)\}_{t=0}^{T-1}$ depends on the inverse of $\{R(t)\}_{t=1}^{T}$. In fact, the feasibility of inverse of $R$ is implied by the expression of $J(\gamma)$ as follows:

$$
\begin{aligned}
J(\gamma)= & \sum_{t=0}^{T}\left[\begin{array}{c}
x \\
w
\end{array}\right]^{\prime}\left[\begin{array}{cc}
A^{\prime} P A+C^{\prime} C-P(t-1) & A^{\prime} P B+C^{\prime} D \\
B^{\prime} P A+D^{\prime} C & -R
\end{array}\right]\left[\begin{array}{l}
x \\
w
\end{array}\right] \\
& +x^{\prime}(0)\left(P(-1)-\gamma^{2} S\right) x(0) .
\end{aligned}
$$

The left of proof is similar to that in the preceding section and thus omitted.

\section{Characterization of $\mathscr{H}_{-}$Index from Frequency Domain}

The preceding sections investigated $\mathscr{H}_{-}$index of linear discrete time-varying systems from time domain. However, some practical faulted systems are time invariant, and the fault detection based on the result from time-invariant may be more effective. Thus it is necessary to investigate the $\mathscr{H}_{-}$ index of linear time-invariant systems. Note that linear timeinvariant systems can be stated as a transfer function. In addition, the $\mathscr{H}_{-}$index in frequency domain can be defined as the minimal singular value over all the frequency range. In this section, we will develop a condition to characterize $\mathscr{H}_{-}$ index in frequency domain for linear discrete time-invariant systems in infinite time horizon.

Let $\mathbf{G}(z)$ be a real rational transfer function matrix of a proper linear discrete time-invariant system with a statespace realization:

$$
\mathbf{G}(z)=C(z I-A)^{-1} B+D=:\left[\begin{array}{c|c}
A & B \\
\hline C & D
\end{array}\right]
$$

where $z$ is the delay operator. Its adjoint system is defined as [30]

$$
\mathbf{G}^{\sim}(z)=B^{\prime}\left(z^{-1} I-A^{\prime}\right)^{-1} C^{\prime}+D^{\prime}
$$

Definition 11 (see [23]). Assume that system $\mathbf{G}$ is linear time invariant and stable (i.e., $A$ is stable). The $\mathscr{H}_{-}$index for discrete time-invariant system $\mathbf{G}$ is defined as

$$
\|\mathbf{G}\|_{-}^{[0, \infty)}:=\inf _{\theta \in[0,2 \pi)^{-}} \underline{\sigma}\left(\mathbf{G}\left(e^{j \theta}\right)\right) .
$$

The above definition is given in frequency domain. However, it can be shown that the above definition is equivalent to the definition in time domain as follows:

$$
\|\mathbf{G}\|_{-}^{[0, \infty)}:=\inf _{w \neq 0} \frac{\|\mathbf{G} w\|_{2}}{\|w\|_{2}}=\inf _{w \neq 0} \frac{\|y\|_{2}}{\|w\|_{2}}, \quad \forall w \in \mathscr{L}_{2}[0, \infty)
$$

The proof is similar to that in $[27,30]$ and thus omitted.

The following theorem characterizes the $\mathscr{H}_{-}$index of linear discrete time-invariant systems. 
Theorem 12. Consider linear discrete time-invariant system G. Let $\gamma$ be a nonnegative scalar; that is, $\gamma \geq 0$. The following conditions are equivalent:

(1) there exists a real symmetric solution $P$ of the following Riccati equation, necessarily unique:

$$
\begin{aligned}
P= & A^{\prime} P A+C^{\prime} C+\left(D^{\prime} C+B^{\prime} P A\right)^{\prime} \\
& \times\left(\gamma^{2} I-D^{\prime} D-B^{\prime} P B\right)^{-1}\left(D^{\prime} C+B^{\prime} P A\right)
\end{aligned}
$$

with $R=\gamma^{2} I-D^{\prime} D-B^{\prime} P B<0$ and $\left|\lambda\left(A_{+}\right)\right|<1$, where $A_{+}=A+B\left(\gamma^{2} I-D^{\prime} D-B^{\prime} P B\right)^{-1}\left(D^{\prime} C+B^{\prime} P A\right)$,

(2) (a) $(A, B)$ is stabilizable;

(b) $\|\mathbf{G}\|_{-}^{[0, \infty)}>\gamma$.

Remark 13. Different from discrete time bounded real lemma [28], whether $P$ is positive or negative definite is not guaranteed here.

Remark 14. It is not necessary to have $D^{\prime} D>\gamma^{2} I$. For example, consider $\mathbf{G}(z)=c(z-a)^{-1} b$ with $|a|<1$ and $d=0$. To do the bilinear transformation $z=(s+1) /(s-1)$ to $\mathbf{G}(z)$, we have $\widehat{\mathbf{G}}(s)=(c b /(a+1))+\left(\left(2 c a b /(1+a)^{2}\right) /(s+((1-a) /(1+a)))\right)$. Thus, $\|\widehat{\mathbf{G}}(z)\|_{-}=\|\mathbf{G}(s)\|_{-}>|c b /(a+1)|>0$.

5.1. Proof of Theorem 12. The proof is based on linear quadratic optimization [31]. Consider the linear quadratic form

$$
J(\gamma)=\sum_{t=0}^{\infty}\left[\begin{array}{l}
x(t) \\
w(t)
\end{array}\right]^{\prime}\left[\begin{array}{ll}
Q & L^{\prime} \\
L & R
\end{array}\right]\left[\begin{array}{c}
x(t) \\
w(t)
\end{array}\right]
$$

subject to

$$
\begin{aligned}
& x(t+1)=A x(t)+B w(t), \\
& x(0)=\xi, \quad \lim _{t \rightarrow \infty} x(t)=0 .
\end{aligned}
$$

Doing $z$-transform for both state space equation and performance index leads to the following new performance index in frequency domain:

$$
J(\gamma, w)=\oint_{|z|=1} \tilde{u}\left(z^{-1}\right) \Psi(z) \widetilde{u}(z) d z
$$

where

$$
\Psi(z)=\left[\begin{array}{c}
\left(z^{-1} I-A\right)^{-1} B \\
I
\end{array}\right]^{\prime}\left[\begin{array}{cc}
Q & L^{\prime} \\
L & R
\end{array}\right]\left[\begin{array}{c}
(z I-A)^{-1} B \\
I
\end{array}\right]
$$

and where $\tilde{u}$ is the $z$-transform of the input sequence $u$. Define

$$
\Psi_{K}(z):=Y^{\prime}\left(z^{-1}\right) \Psi(z) Y(z),
$$

where $Y(z)=I-K\left(z I-A_{K}\right)^{-1} B$ and $A_{K}=A-B K$.

We have the following lemma from [31], stating that the existence of the spectral factorization of $\Psi_{K}$ can be characterized as an algebraic Riccati equation.
Lemma 15 (see [31]). The following conditions are equivalent.

(1) there exists a real symmetric solution $P$ of the following Riccati equation, necessarily unique:

$$
P=A^{\prime} P A+Q-\left(L+B^{\prime} P A\right)^{\prime}\left(R+B^{\prime} P B\right)^{-1}\left(L+B^{\prime} P A\right)
$$

with $R+B^{\prime} P B>0$ and $\left|\lambda\left(A_{+}\right)\right|<1$, where $A_{+}=$ $A-B\left(R+B^{\prime} P B\right)^{-1}\left(L+B^{\prime} P A\right)$,

(2) (a) $(A, B)$ is stabilizable;

(b) for some (and hence all) $K$ such that $\left|\lambda\left(A_{K}\right)\right| \neq 1$, $\Psi_{K}(z)>0$ for all $|z|=1$,

(3) (a) $(A, B)$ is stabilizable;

(b) for some (and hence all) $K, \Psi_{K}(z)>0$ for some (and hence all) $|z|=1$ such that $|z|=1$ and $\lambda\left(A_{K}\right) \neq z$.

The proof of Theorem 12 is as follows.

Proof. It has been shown that $\|\mathbf{G}\|_{-}^{[0, \infty)}>\gamma$ can be characterized as the following performance index:

$$
\begin{aligned}
J(\gamma) & =\sum_{t=0}^{\infty}\left[(C x+D w)^{\prime}(C x+D w)-\gamma^{2} w^{\prime} w\right] \\
& =\sum_{t=0}^{\infty}\left[\begin{array}{c}
x(t) \\
w(t)
\end{array}\right]^{\prime}\left[\begin{array}{cc}
C^{\prime} C & C^{\prime} D \\
D^{\prime} C & D^{\prime} D-\gamma^{2} I
\end{array}\right]\left[\begin{array}{c}
x(t) \\
w(t)
\end{array}\right]
\end{aligned}
$$

subject to

$$
\begin{aligned}
& x(t+1)=A x(t)+B w(t), \\
& x(0)=0, \quad \lim _{t \rightarrow \infty} x(t)=0 .
\end{aligned}
$$

To apply Lemma 15 to the above performance index (39), we have Theorem 12.

5.2. Riccati and Inequality Condition. In this subsection, we will further characterize the $\mathscr{H}_{-}$index of linear discrete timeinvariant systems in terms of Riccati and inequality, which is more suitable for fault detection application. The main result is as follows.

Theorem 16. Assume that the linear discrete time-invariant system $\mathbf{G}$ is stable (i.e., $A$ is stable). Let $\gamma$ be a nonnegative scalar; that is, $\gamma \geq 0$. The following conditions are equivalent:

(1) $\|\mathbf{G}\|_{-}^{[0, \infty)}>\gamma$,

(2) there exists a real symmetric solution $P$ of the following Riccati equation:

$$
\begin{aligned}
P= & A^{\prime} P A+C^{\prime} C+\left(D^{\prime} C+B^{\prime} P A\right)^{\prime} \\
& \times\left(\gamma^{2} I-D^{\prime} D-B^{\prime} P B\right)^{-1}\left(D^{\prime} C+B^{\prime} P A\right)
\end{aligned}
$$

with $R=\gamma^{2} I-D^{\prime} D-B^{\prime} P B<0$ and $A_{+}=A+B\left(\gamma^{2} I-\right.$ $\left.D^{\prime} D-B^{\prime} P B\right)^{-1}\left(D^{\prime} C+B^{\prime} P A\right)$ has no eigenvalues on the unit circle, 
(3) there exists a symmetric matrix $P$ such that

$$
\begin{aligned}
A^{\prime} P A- & P+C^{\prime} C+\left(D^{\prime} C+B^{\prime} P A\right)^{\prime} \\
& \times\left(\gamma^{2} I-D^{\prime} D-B^{\prime} P B\right)^{-1}\left(D^{\prime} C+B^{\prime} P A\right)
\end{aligned}
$$

$>0$,

(4) there exists a symmetric matrix $P$ such that

$$
\left[\begin{array}{cc}
A^{\prime} P A-P+C^{\prime} C & A^{\prime} P B+C^{\prime} D \\
B^{\prime} P A+D^{\prime} C & -R
\end{array}\right]>0 .
$$

Proof. Condition (1) implies Condition (2) by Theorem 12. define

To show that Condition (2) implies Condition (1), we

$$
\mathbf{W}:=\left[\begin{array}{c|c}
A & B \\
\hline-R^{-1}\left(D^{\prime} C+B^{\prime} P A\right) & I
\end{array}\right] .
$$

By the Riccati equation (41), we have

$$
\begin{aligned}
\left(z^{-1} I-\right. & \left.A^{\prime}\right) P(z I-A)+\left(z^{-1} I-A^{\prime}\right) P A+A^{\prime} P(z I-A) \\
= & P-A^{\prime} P A \\
= & C^{\prime} C+\left(D^{\prime} C+B^{\prime} P A\right)^{\prime}\left(\gamma^{2} I-D^{\prime} D-B^{\prime} P B\right)^{-1} \\
& \times\left(D^{\prime} C+B^{\prime} P A\right) .
\end{aligned}
$$

Now premultiply the above equation by $B^{\prime}\left(z^{-1} I-A^{\prime}\right)^{-1}$ and postmultiply it by $(z I-A)^{-1} B$ to get

$$
\begin{aligned}
B^{\prime} P B & +B^{\prime} P A(z I-A)^{-1} B+B^{\prime}\left(z^{-1} I-A^{\prime}\right)^{-1} A^{\prime} P B \\
= & B^{\prime}\left(z^{-1} I-A^{\prime}\right)^{-1} C^{\prime} C(z I-A)^{-1} B \\
& +B^{\prime}\left(z^{-1} I-A^{\prime}\right)^{-1}\left(D^{\prime} C+B^{\prime} P A\right)^{\prime} \\
& \times\left(\gamma^{2} I-D^{\prime} D-B^{\prime} P B\right)^{-1} \\
& \times\left(D^{\prime} C+B^{\prime} P A\right)(z I-A)^{-1} B .
\end{aligned}
$$

Using equality (46), we have the following derivations:

$$
\begin{aligned}
& \mathbf{G}^{\sim} \mathbf{G}-\mathbf{W}^{\sim}(-R) \mathbf{W} \\
& =\left[B^{\prime}\left(z^{-1} I-A^{\prime}\right)^{-1} C^{\prime}+D^{\prime}\right]\left[C(z I-A)^{-1} B+D\right] \\
& +\left[B^{\prime}\left(z^{-1} I-A^{\prime}\right)^{-1}\left(-R^{-1}\left(D^{\prime} C+B^{\prime} P A\right)\right)^{\prime}+I\right] \\
& \times R\left[-R^{-1}\left(D^{\prime} C+B^{\prime} P A\right)(z I-A)^{-1} B+I\right] \\
& =D^{\prime} D+D^{\prime} C(z I-A)^{-1} B+B^{\prime}\left(z^{-1} I-A\right)^{-1} C^{\prime} D \\
& +B^{\prime}\left(z^{-1} I-A^{\prime}\right)^{-1} C^{\prime} C(z I-A)^{-1} B \\
& +B^{\prime}\left(z^{-1} I-A^{\prime}\right)^{-1}\left[-R^{-1}\left(D^{\prime} C+B^{\prime} P A\right)\right]^{\prime} \\
& \times R\left[-R^{-1}\left(D^{\prime} C+B^{\prime} P A\right)\right](z I-A)^{-1} B \\
& +B^{\prime}\left(z^{-1} I-A^{\prime}\right)^{-1}\left[-R^{-1}\left(D^{\prime} C+B^{\prime} P A\right)\right]^{\prime} R \\
& +R\left[-R^{-1}\left(D^{\prime} C+B^{\prime} P A\right)\right](z I-A)^{-1} B+R \\
& =D^{\prime} D+D^{\prime} C(z I-A)^{-1} B+B^{\prime}\left(z^{-1} I-A^{\prime}\right)^{-1} C^{\prime} D \\
& +B^{\prime}\left(z^{-1} I-A^{\prime}\right)^{-1} C^{\prime} C(z I-A)^{-1} B \\
& +B^{\prime}\left(z^{-1} I-A^{\prime}\right)^{-1}\left(D^{\prime} C+B^{\prime} P A\right)^{\prime} R^{-1} \\
& \times\left(D^{\prime} C+B^{\prime} P A\right)(z I-A)^{-1} B \\
& -B^{\prime}\left(z^{-1} I-A^{\prime}\right)^{-1}\left(D^{\prime} C+B^{\prime} P A\right)^{\prime} \\
& -\left(D^{\prime} C+B^{\prime} P A\right)(z I-A)^{-1} B+R \\
& =D^{\prime} D+B^{\prime}\left(z^{-1} I-A^{\prime}\right)^{-1} C^{\prime} C(z I-A)^{-1} B \\
& -B^{\prime}\left(z^{-1} I-A^{\prime}\right)^{-1}\left(B^{\prime} P A\right)^{\prime}-B^{\prime} P A(z I-A)^{-1} B \\
& +B^{\prime}\left(z^{-1} I-A^{\prime}\right)^{-1}\left(D^{\prime} C+B^{\prime} P A\right)^{\prime} R^{-1} \\
& \times\left(D^{\prime} C+B^{\prime} P A\right)(z I-A)^{-1} B+\gamma^{2} I-D^{\prime} D-B^{\prime} P B \\
& =D^{\prime} D-B^{\prime}\left(z^{-1} I-A^{\prime}\right)^{-1}\left(B^{\prime} P A\right)^{\prime} \\
& -B^{\prime} P A\left(z I-A^{\prime}\right)^{-1} B+B^{\prime} P B \\
& +B^{\prime} P A\left(z I-A^{\prime}\right)^{-1} B+B^{\prime}(z I-A)^{-1} A^{\prime} P B \\
& +\gamma^{2} I-D^{\prime} D-B^{\prime} P B \\
& =\gamma^{2} I \text {. }
\end{aligned}
$$

That is,

$$
\mathbf{G}^{\sim} \mathbf{G}-\gamma^{2} I=\mathbf{W}^{\sim}(-R) \mathbf{W} .
$$


Thus, $R<0$ implies $\mathbf{G}^{\sim} \mathbf{G}-\gamma^{2} I \geq 0$. Since

$$
\mathbf{W}^{-1}=\left[\begin{array}{c|c}
A+B R^{-1}\left(D^{\prime} C+B^{\prime} P A\right) & B \\
\hline R^{-1}\left(D^{\prime} C+B^{\prime} P A\right) & I
\end{array}\right]
$$

exists, we have $\mathbf{G}^{\sim} \mathbf{G}-\gamma^{2} I>0$, and thus $\|\mathbf{G}\|_{-}^{[0, \infty)}>\gamma$.

To show that Condition (3) implies Condition (1), by using the Riccati inequality (42) we have

$$
\mathbf{G}^{\sim} \mathbf{G}-\gamma^{2} I>\mathbf{W}^{\sim}(-R) \mathbf{W} .
$$

Since $R<0$, we have $\mathbf{G}^{\sim} \mathbf{G}-\gamma^{2} I>0$ and thus $\|\mathbf{G}\|_{-}^{[0, \infty)}>\gamma$.

To show that Condition (1) implies Condition (3), we consider the following system $\widehat{\mathbf{G}}$ :

$$
\widehat{\mathbf{G}}:=\left[\begin{array}{c|cc}
A & B & 0 \\
\hline C & D & 0 \\
\epsilon_{1} I & 0 & \left(\gamma+\epsilon_{2}\right) I
\end{array}\right]
$$

which is also square or tall. Then there exist two positive small scalars $\epsilon_{1}$ and $\epsilon_{2}$ such that $\|\mathbf{G}\|_{-}^{[0, \infty)}>\gamma$. Applying Condition (2) for $\widehat{\mathbf{G}}$ provides

$$
\begin{aligned}
& P=A^{\prime} P A+C^{\prime} C+\epsilon_{1}^{2} I \\
& -\left[\begin{array}{c}
D^{\prime} C+B^{\prime} P A \\
\left(\gamma+\epsilon_{2}\right) \epsilon_{1} I
\end{array}\right]^{\prime} \\
& \times\left[\begin{array}{cc}
\gamma^{2} I-D^{\prime} D-B^{\prime} P B & 0 \\
0 & {\left[\left(\gamma+\epsilon_{2}\right)^{2}-\gamma^{2}\right] I}
\end{array}\right]^{-1} \\
& \times\left[\begin{array}{c}
D^{\prime} C+B^{\prime} P A \\
\left(\gamma+\epsilon_{2}\right) \epsilon_{1} I
\end{array}\right] .
\end{aligned}
$$

Expansion leads to

$$
\begin{aligned}
A^{\prime} P A- & P+C^{\prime} C+\left(D^{\prime} C+B^{\prime} P A\right)^{\prime}\left(\gamma^{2} I-D^{\prime} D-B^{\prime} P B\right)^{-1} \\
& \times\left(D^{\prime} C+B^{\prime} P A\right) \\
= & -\epsilon_{1}^{2} I+\epsilon_{1}^{2}\left(\gamma+\epsilon_{2}\right)^{2}\left(2 \epsilon_{2} \gamma+\epsilon_{2}^{2}\right)^{-1} I \\
= & \gamma^{2} \epsilon_{1}^{2}\left(2 \epsilon_{2} \gamma+\epsilon_{2}^{2}\right)^{-1} I>0 .
\end{aligned}
$$

So we have Condition (3).

The equivalence between Condition (3) and Condition (4) is by Schur complement [32].

Remark 17. The condition (43) is consistent with that in [33]. In [33], a general LMI condition is proposed to measure the system gain in frequency domain in form of general KYP lemma. However, as to $\mathscr{H}_{-}$index, Theorems 12 and 16 provide more rich conditions, so that they can be applied to more cases.
Inequality (43) is an LMI in terms of $P$, which is numerically solvable [32]. In addition, we can have the following optimization to compute $\|\mathbf{G}\|_{-}^{[0, \infty)}$ :

$$
\max _{P, \alpha>0} \gamma=\sqrt{\alpha}, \quad \text { s.t. LMI (43). }
$$

The following result is for square systems.

Corollary 18. Assume that the linear discrete time-invariant system $\mathbf{G}$ is stable (i.e., $A$ is stable) and square. Let $\gamma$ be a nonnegative scalar; that is, $\gamma \geq 0$. The following conditions are equivalent:

(1) $\|\mathbf{G}\|_{-}^{[0, \infty)}>\gamma$,

(2) there exists a real symmetric solution $P$ of the following Riccati equation:

$$
\begin{aligned}
P= & A P A^{\prime}+B B^{\prime}+\left(D B^{\prime}+C P A^{\prime}\right)^{\prime} \\
& \times\left(\gamma^{2} I-D D^{\prime}-C P C^{\prime}\right)^{-1}\left(D B^{\prime}+C P A^{\prime}\right)
\end{aligned}
$$

with $R=\gamma^{2} I-D D^{\prime}-C P C^{\prime}<0$ and $A_{+}=A^{\prime}+C^{\prime}\left(\gamma^{2} I-\right.$ $\left.D D^{\prime}-C P C^{\prime}\right)^{-1}\left(D B^{\prime}+C P A^{\prime}\right)$ has no eigenvalues on the unit circle,

(3) there exists a symmetric matrix $P$ such that

$$
\begin{aligned}
A P A^{\prime}- & P+B B^{\prime}+\left(D B^{\prime}+C P A^{\prime}\right)^{\prime} \\
& \times\left(\gamma^{2} I-D D^{\prime}-C P C^{\prime}\right)^{-1}\left(D B^{\prime}+C P A^{\prime}\right) \\
> & 0
\end{aligned}
$$

(4) there exists a symmetric matrix $P$ such that

$$
\left[\begin{array}{cc}
A P A^{\prime}-P+B B^{\prime} & B D^{\prime}+A P C^{\prime} \\
D B^{\prime}+C P A^{\prime} & -R
\end{array}\right]>0
$$

Proof. The proof is based on the result for adjoint system of square system, $\|\mathbf{G}\|_{-}=\left\|\mathbf{G}^{\sim}\right\|_{-}$.

\section{Comparison with Bounded Real Lemma}

Bounded real lemma is a very important condition to characterize $\mathscr{H}_{\infty}$ norm in control field [28-30]. [28, 29] have derived the condition for bounded real lemma of discretetime systems. Our results show that the $\mathscr{H}_{-}$index is not dual to $\mathscr{H}_{\infty}$ norm, but with some discrepancies, which are stated as follows.

(i) Our result on $\mathscr{H}_{-}$index generally is only for tall or square systems. The reason is that for wide systems, $\mathscr{H}_{\text {_ }}$ index is generally zero. However, bounded real lemma for $\mathscr{H}_{\infty}$ norm is also applicable to all kinds of systems, including wide systems.

(ii) They end up with the same difference Riccati equation (3). When restricted to linear time-invariant systems, they end up with the same algebraic Riccati equation (41). 
(iii) The constraint associated with bounded real lemma is positive $(R>0)$, while the constraint associated with $\mathscr{H}_{\text {- }}$ index is negative $(R<0)$.

(iv) The solution for $\mathscr{H}_{-}$index is not necessarily positive, while the solution associated with bounded real lemma is positive $(P>0)$.

(v) For linear discrete time-invariant systems, they lead to the same inequality but with opposite sign (inequality (43)).

\section{Examples}

In this section, we use four examples to demonstrate our results. Examples 19-21 are to verify our results, while Example 22 is to demonstrate its application in fault detection.

Example 19. Consider linear discrete time-varying system G with the following coefficients:

$$
\begin{gathered}
A=\left[\begin{array}{cc}
0.4 & 0.2 \times 2^{(3-t)} \\
0 & 0.2
\end{array}\right], \quad B=\left[\begin{array}{ll}
0.2 & 0.3 \\
0.4 & 0.4
\end{array}\right], \\
C=\left[\begin{array}{cc}
0.1 & 0.5 \\
0.3 & 0.4 \\
3 & 2
\end{array}\right], \quad D=\left[\begin{array}{cc}
3 & 4 \\
1 & 5 \\
3 & 2-0.2 \times 2^{(3-t)}
\end{array}\right] .
\end{gathered}
$$

Let the initial state $x_{0}=0$. Choose $\gamma=1.5$ and $T=20$. Figure 1 shows $R$ along with step $t$. We solve the Riccati equation (3) iteratively. It can be seen that $R(t)$ is negative, implying that the inequality constraint $R(t)<0$ is satisfied. The solution $P(t)$ of the Riccati equation (3) exists, whose eigenvalues are shown in Figure 2. It can be seen that $P(t)$ is not necessarily negative or positive. It is also not monotonic. In addition, it fails to converge into the $P$ of the LTI case in which time-varying terms are removed in system coefficients; that is,

$$
P_{\text {LTI }}=\left[\begin{array}{cc}
1.1498 & 0.2702 \\
0.2702 & -0.0321
\end{array}\right]
$$

Example 20. This example is to demonstrate the result for unknown initial states. Consider the same system in Example 19, but with unknown initial state $x_{0}$. We choose $P(20)=\operatorname{diag}(1,1), S(-1)=0$, and $\gamma=0.5$ for modified $\mathscr{H}$ _ index (Definition 6). We solve the Riccati equation (18) iteratively. Figure 3 shows $R$ along with step $t$. It can be seen that $R(t)$ is negative, implying that the inequality constraint $R(t)<0$ is satisfied. The solution of the Riccati equation (18) $P(t)$ exists, whose eigenvalues are shown in Figure 4. It can be seen that it is not necessarily negative or positive. It is also not monotonic. In addition, it fails to converge into the $P$ of the LTI case in which time-varying terms are removed in system coefficients:

$$
P_{\mathrm{LTI}}=\left[\begin{array}{ll}
3.4125 & 1.6837 \\
1.6837 & 0.9239
\end{array}\right]
$$

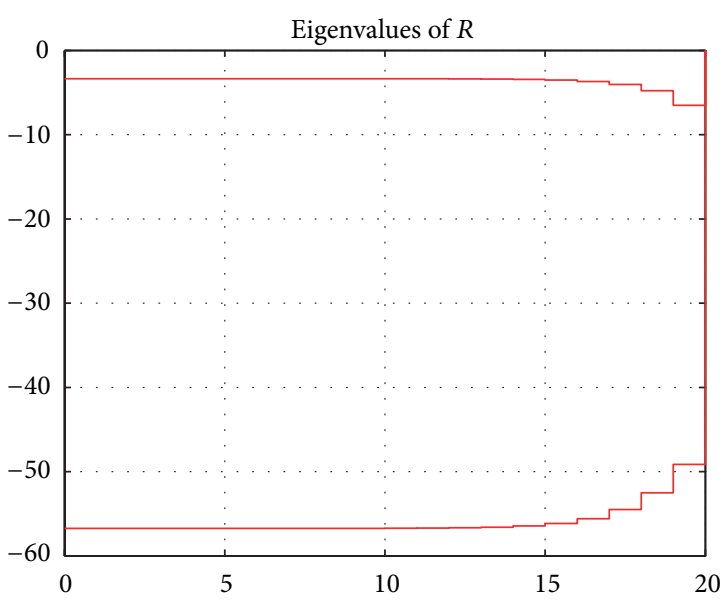

FIgURE 1: Eigenvalues of $R$ versus time $t$, Example 19.

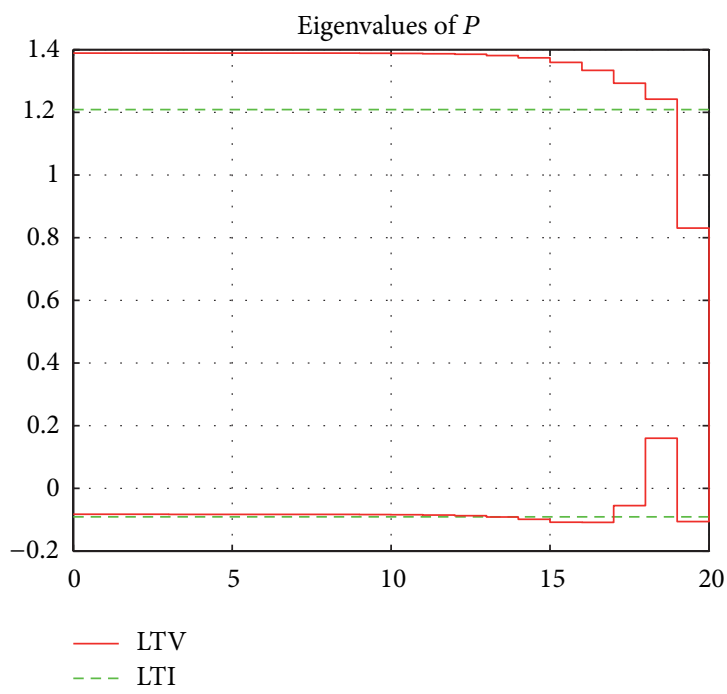

Figure 2: Eigenvalues of $P$ versus time $t$, Example 19.

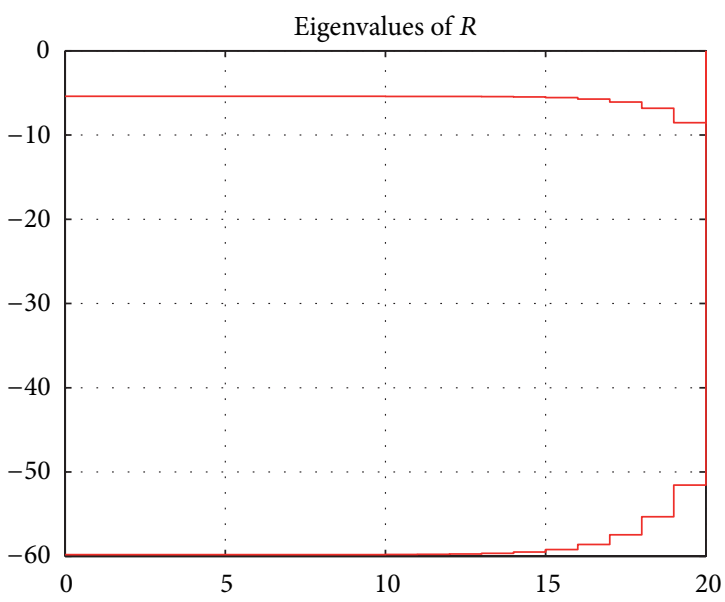

FIgURE 3: Eigenvalues of $R$ versus time $t$, Example 20. 


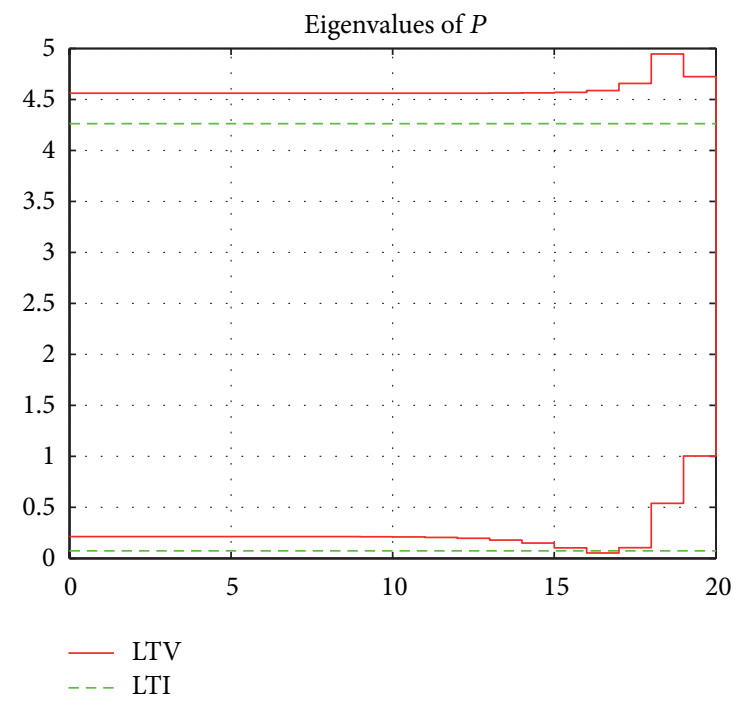

Figure 4: Eigenvalues of $P$ versus time $t$, Example 20.

Example 21. This example is to demonstrate the result for linear time-invariant systems. Consider linear discrete time-invariant system $\mathbf{G}$ which has time varying terms in Example 19 removed. Let $\gamma=1.5$. Since system $\mathbf{G}$ is linear time-invariant, Theorem 16 is applicable, so we solve LMI (43) to obtain

$$
P=\left[\begin{array}{cc}
-43.8243 & 5.4034 \\
5.4034 & -40.5231
\end{array}\right]
$$

The eigenvalues of $P$ are $\lambda=-47.8236,-36.5238<0$, implying that it is negative. Thus, we have $\|\mathbf{G}\|_{-}^{[0, \infty)}>1.5$. This just gives a lower bound of $\mathscr{H}_{-}$index. As LMI is numerically solvable, we can solve the optimization problem (54) to obtain that $\|\mathbf{G}\|_{-}^{[0, \infty)}=2.1426$.

Example 22. Our result on $\mathscr{H}_{-}$index is very useful in fault detection for evaluating the fault detection ability. To demonstrate it, we consider the following faulted system G:

$$
\begin{gathered}
A=\left[\begin{array}{cc}
-0.08 & -2 \times e^{(50-t) / 100} \\
0 & -0.02
\end{array}\right], \quad B=\left[\begin{array}{cc}
1 & 3 \\
0.4 & 1
\end{array}\right], \\
C=\left[\begin{array}{cc}
0.1 & 0.8 \\
-0.3 & -0.4 \\
0.4 & 0.5
\end{array}\right], \quad D=\left[\begin{array}{cc}
-5 & 3 \\
1 & 3 \\
2 & 9-e^{(50-t) / 100}
\end{array}\right] .
\end{gathered}
$$

This system can be thought of as a system from fault signal to output signal. In other words, this system represents the fault dynamics. Now we consider to implement two filters $\mathbf{F}_{1}$ and $\mathbf{F}_{2}$. We assume that the inputs for the two filters are the output of system $\mathbf{G}$, while the outputs of the two filters

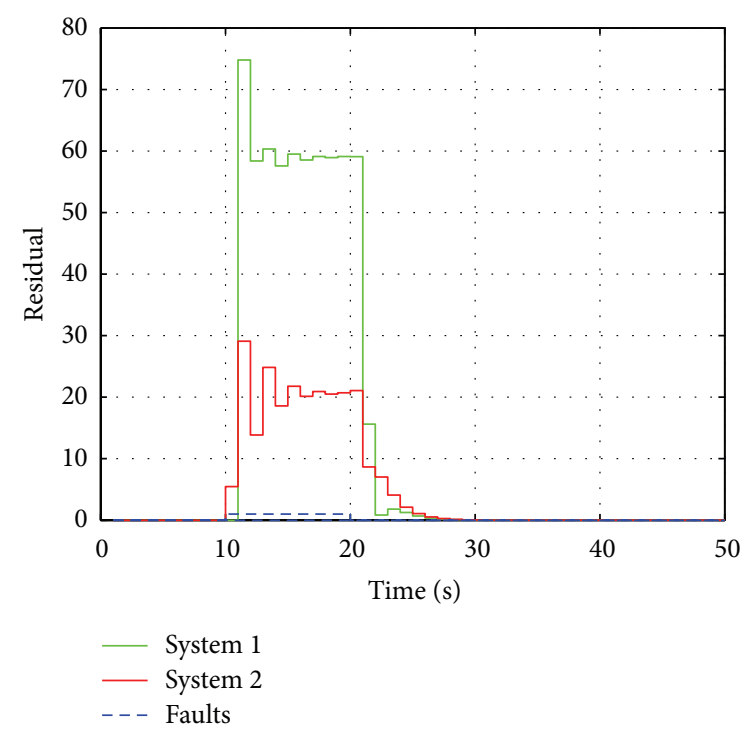

FIGURE 5: Fault detection comparison.

are their residual signals, which are used for fault diagnosis purpose. $\mathbf{F}_{1}$ is assumed with the following coefficients:

$$
\begin{array}{cc}
\widehat{A}=\left[\begin{array}{cc}
-0.1 & 0 \\
0 & -0.5
\end{array}\right], \quad \widehat{B}=\left[\begin{array}{lll}
1 & 3 & 9 \\
0 & 9 & 9
\end{array}\right], \\
\widehat{C}=\left[\begin{array}{ll}
1 & 1 \\
1 & 1 \\
1 & 1
\end{array}\right], & \widehat{D}=\left[\begin{array}{lll}
1 & 0 & 0 \\
0 & 1 & 0 \\
0 & 0 & 1
\end{array}\right] .
\end{array}
$$

$\mathbf{F}_{2}$ is the same as $\mathbf{F}_{1}$, except

$$
\widehat{B}=\left[\begin{array}{lll}
1 & 3 & 3 \\
0 & 9 & 2
\end{array}\right] .
$$

We compare the two filters by comparing their fault sensitivity from fault to residual. In other words, we compare the $\mathscr{H}_{-}$indices of two systems: $\mathbf{F}_{1} \mathbf{G}$ and $\mathbf{F}_{2} \mathbf{G}$. We choose the time length as $(0,50)$. We can use Theorem 3 to obtain the lower bound of the $\mathscr{H}_{-}$indices. Through trial and error, we can further obtain their values; that is, $\left\|\mathbf{F}_{1} \mathbf{G}\right\|_{-,[0,50]}=8.5$ and $\left\|\mathbf{F}_{2} \mathbf{G}\right\|_{-,[0,50]}=2.5$. It means that filter $\mathbf{F}_{1}$ implies higher fault detection ability. To demonstrate it, we run the systems at the same fault signal. The fault is chosen as

$$
f(t)= \begin{cases}{\left[\begin{array}{l}
1 \\
0
\end{array}\right],} & 10 \leq t \leq 20 \\
0, & \text { elsewhere. }\end{cases}
$$

It implies that fault occurs at $10 \mathrm{~s}$. Figure 5 shows the residuals along with time under the two filters. It can be seen that when no fault happens, the residual shows no gain, implying no fault is diagnosed. When fault happens between $10 \mathrm{~s}$ and $20 \mathrm{~s}$, the residual signal jumps rapidly, which implies that the fault is presented. Furthermore, it is obvious that filter $\mathbf{F}_{1}$ triggers high variations than filter $\mathbf{F}_{2}$ and thus implies better fault detection ability in terms of sensitivity. 
From this example, it can be seen that our proposed characterization of $\mathscr{H}_{-}$index has the ability of evaluating the system sensitivity in fault detection. It can provide guide for choosing the parameters in fault detection filter. Specifically, it may be possible to optimize certain parameters in the filter to seek for the best fault detection ability in terms of $\mathscr{H}_{-}$ index. The detailed optimization algorithm is out of scope of this paper.

\section{Conclusion}

This paper characterizes the $\mathscr{H}_{\text {_ }}$ index of linear discrete time systems. At first, we developed a sufficient and necessary condition for the $\mathscr{H}_{-}$index of linear discrete time-varying systems in finite time horizon. The condition is characterized as the existence of solution to a certain difference Riccati equation with an inequality constraint. The result has been further extended to systems with unknown initial condition based on modified $\mathscr{H}_{-}$index. From the perspective of frequency domain, we developed a similar condition in terms of an algebraic Riccati equation with an inequality condition for linear discrete time-invariant systems. To compare it with the famous bounded real lemma, it can be seen that $\mathscr{H}_{-}$ index is not completely dual to $\mathscr{H}_{\infty}$ norm. Several examples were given to illustrate our results. In particular, one example demonstrated its application in fault detection. However, the direct fault detection filter design inspired by our proposed result is still missing and very desirable.

\section{Acknowledgment}

This work is supported by the National Sciences and Engineering Research Council (NSERC) of Canada.

\section{References}

[1] J. Chen and R. J. Patton, Robust Model-Based Fault Diagnosis for Dynamic Systems, Kluwer Academic Publishers, Boston, Mass, USA, 1999.

[2] P. M. Frank, "Fault diagnosis in dynamic systems using analytical and knowledge-based redundancy. A survey and some new results," Automatica, vol. 26, no. 3, pp. 459-474, 1990.

[3] P. M. Frank and X. Ding, "Frequency domain approach to optimally robust residual generation and evaluation for modelbased fault diagnosis," Automatica, vol. 30, no. 5, pp. 789-804, 1994.

[4] R. J. Patton, "Robustness in model-based fault diagnosis: the 1995 situation," IFAC Annual Reviews in Control, vol. 21, pp. 103123, 1997.

[5] P. M. Frank and X. Ding, "Survey of robust residual generation and evaluation methods in observer-based fault detection systems," Journal of Process Control, vol. 7, no. 6, pp. 403-424, 1997.

[6] A. Casavola, D. Famularo, and G. Franzè, "A robust deconvolution scheme for fault detection and isolation of uncertain linear systems: an LMI approach," Automatica, vol. 41, no. 8, pp. 14631472, 2005.

[7] S. X. Ding, P. M. Frank, E. L. Ding, and T. Jeinsch, "Fault detection system design based on a new trade-off strategy," in
Proceedings of the 39th IEEE Confernce on Decision and Control, pp. 4144-4149, Sydney, Australia, December 2000.

[8] D. Henry and A. Zolghadri, "Design of fault diagnosis filters: a multi-objective approach," Journal of the Franklin Institute, vol. 342, no. 4, pp. 421-446, 2005.

[9] M. J. Khosrowjerdi, R. Nikoukhah, and N. Safari-Shad, "Fault detection in a mixed $\mathscr{H}_{2} / \mathscr{H}_{\infty}$ setting," IEEE Transactions on Automatic Control, vol. 50, no. 7, pp. 1063-1068, 2005.

[10] M. J. Khosrowjerdi, R. Nikoukhah, and N. Safari-Shad, "A mixed $\mathscr{H}_{2} / \mathscr{H}_{\infty}$ approach to simultaneous fault detection and control," Automatica, vol. 40, no. 2, pp. 261-267, 2004.

[11] M. L. Rank and H. Niemann, "Norm based design of fault detectors," International Journal of Control, vol. 72, no. 9, pp. 773-783, 1999.

[12] S. X. Ding, T. Jeinsch, P. M. Frank, and E. L. Ding, "A unified approach to the optimization of fault detection systems," International Journal of Adaptive Control and Signal Processing, vol. 14, no. 7, pp. 725-745, 2000.

[13] M. Hou and R. J. Patton, "LMI approach to $\mathscr{H}_{-} / \mathscr{H}_{\infty}$ fault detection observers," in Proceedings of the UKACC International Conference on Control, pp. 305-310, September 1996.

[14] Z. Li, E. Mazars, and I. Jaimoukha, "State space solution to the $\mathscr{H}_{-} / \mathscr{H}_{\infty}$ fault detection problem," in Proceedings of the 45th IEEE Conference on Decision and Control (CDC '06), pp. 21772182, San Diego, Calif, USA, December 2006.

[15] I. M. Jaimoukha, Z. Li, and V. Papakos, "A matrix factorization solution to the $\mathscr{H}_{-} / \mathscr{H} \infty$ fault detection problem," Automatica, vol. 42, no. 11, pp. 1907-1912, 2006.

[16] J. Liu, J. L. Wang, and G.-H. Yang, "An LMI approach to minimum sensitivity analysis with application to fault detection," Automatica, vol. 41, no. 11, pp. 1995-2004, 2005.

[17] F. Tao and Q. Zhao, "Fault detection observer design with unknown inputs," in Proceeding of the IEEE Conference on Control Applications, pp. 1275-1280, Toronto, Canada, August 2005.

[18] J. L. Wang, G.-H. Yang, and J. Liu, "An LMI approach to $\mathscr{H}_{-}$ index and mixed $\mathscr{H}_{-} / \mathscr{H}_{\infty}$ fault detection observer design," Automatica, vol. 43, no. 9, pp. 1656-1665, 2007.

[19] H. B. Wang, L. Lam, S. X. Ding, and M. Y. Zhong, "Iterative linear matrix inequality algorithms for fault detection with unknown inputs," Journal of Systems and Control Engineering, vol. 219, no. 2, pp. 161-172, 2005.

[20] X. Li and K. Zhou, "Fault detection for linear discretetime invariant systems with decoupling and optimization," in Proceedings of the 48th IEEE Conference on Decision and Control Held Jointly with the 28th Chinese Control Conference (CDC/CCC '09), pp. 990-995, Shanghai, China, December 2009.

[21] X. Li and K. Zhou, "A time domain approach to robust fault detection of linear time-varying systems," Automatica, vol. 45, no. 1, pp. 94-102, 2009.

[22] N. Liu and K. Zhou, "Optimal solutions to multi-objective robust fault detection problems," in Proceedings of the 46th IEEE Conference on Decision and Control (CDC '07), pp. 981-988, New Orleans, La, USA, December 2007.

[23] N. Liu and K. Zhou, "Optimal robust fault detection for linear discrete time systems," Journal of Control Science and Engineering, vol. 2008, Article ID 829459, 6 pages, 2008.

[24] X. Li, S. F. Mo, and K. Zhou, "Fault detection for linear discrete time-varying systems," in Proceedings of the 49th IEEE Conference on Decision and Control (CDC '10), pp. 762-767, Atlanta, Ga, USA, December 2010. 
[25] M. Zhong, S. X. Ding, and E. L. Ding, "Optimal fault detection for linear discrete time-varying systems," Automatica, vol. 46, no. 8, pp. 1395-1400, 2010.

[26] X. Li and H. H. T. Liu, "A necessary and sufficient condition for

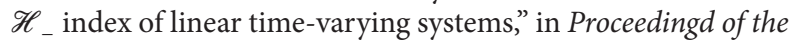
49th IEEE Conference on Decision and Control (CDC '10), pp. 4393-4398, Atlanta, Ga, USA, December 2010.

[27] X. Li and H. H. T. Liu, "Characterization of $\mathscr{H}_{-}$index for linear time-varying systems," Automatica. In press.

[28] C. E. de Souza and L. Xie, "On the discrete-time bounded real lemma with application in the characterization of static state feedback $\mathscr{H}_{\infty}$ controllers," Systems \& Control Letters, vol. 18, no. 1, pp. 61-71, 1992.

[29] K. Furuta and S. Phoojaruenchanachai, "An algebraic approach to discrete-time $\mathscr{H}_{\infty}$ control problems," in Proceedings of the American Control Conference, pp. 3067-3072, San Diego, Calif, USA, May 1990.

[30] K. Zhou, J. C. Doyle, and K. Clover, Robust and Optimal Control, Prentice Hall, Upper Saddle River, NJ, USA, 1996.

[31] B. P. Molinari, "The stabilizing solution of the discrete algebraic Riccati equation," IEEE Transactions on Automatic Control, vol. 20, pp. 396-399, 1975.

[32] S. Boyd, L. El Ghaoui, E. Feron, and V. Balakrishnan, Linear Matrix Inequalities in System and Control Theory, vol. 15 of SIAM Studies in Applied Mathematics, SIAM, Philadelphia, Pa, USA, 1994.

[33] T. Iwasaki, S. Hara, and H. Yamauchi, "Dynamical system design from a control perspective: finite frequency positiverealness approach," IEEE Transactions on Automatic Control, vol. 48, no. 8, pp. 1337-1354, 2003. 


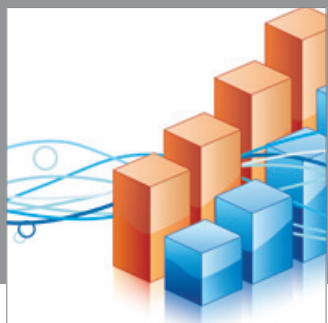

Advances in

Operations Research

mansans

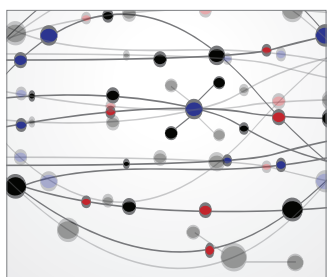

The Scientific World Journal
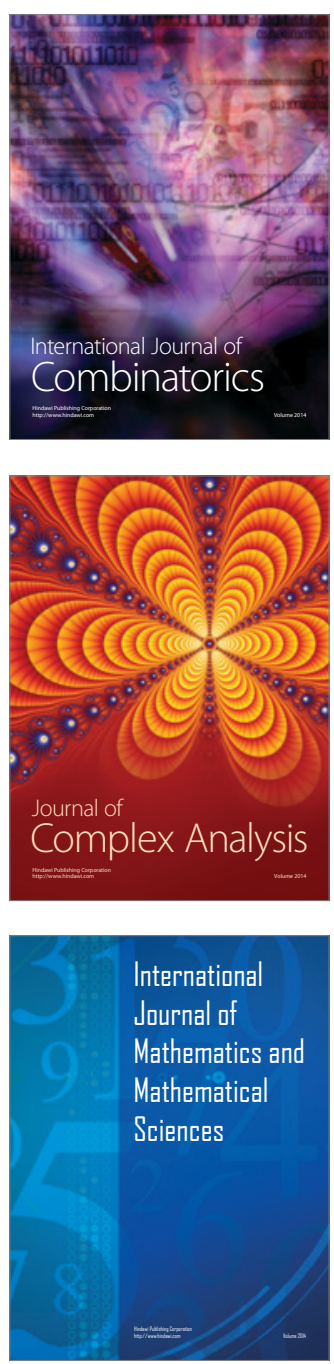
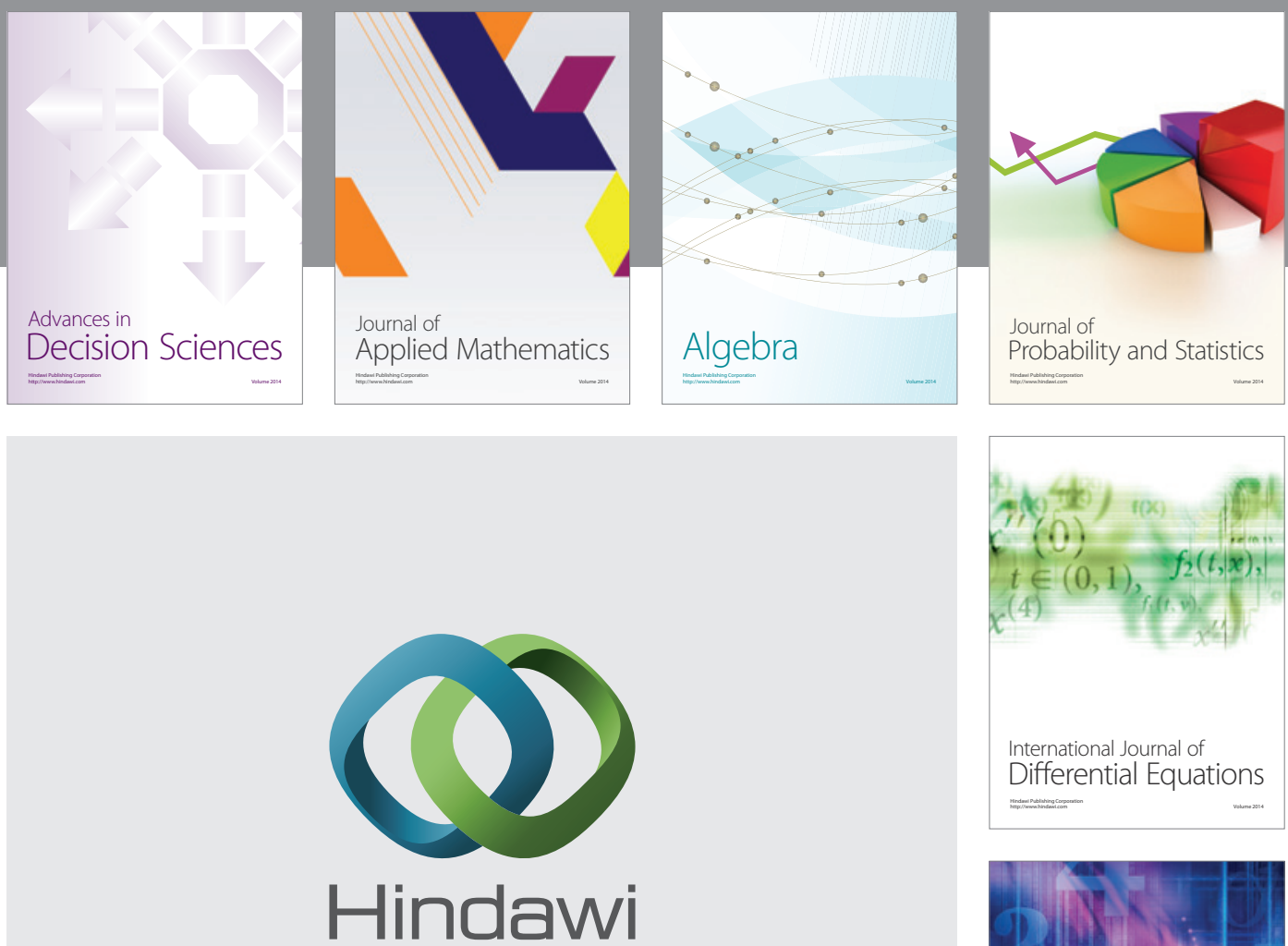

Submit your manuscripts at http://www.hindawi.com
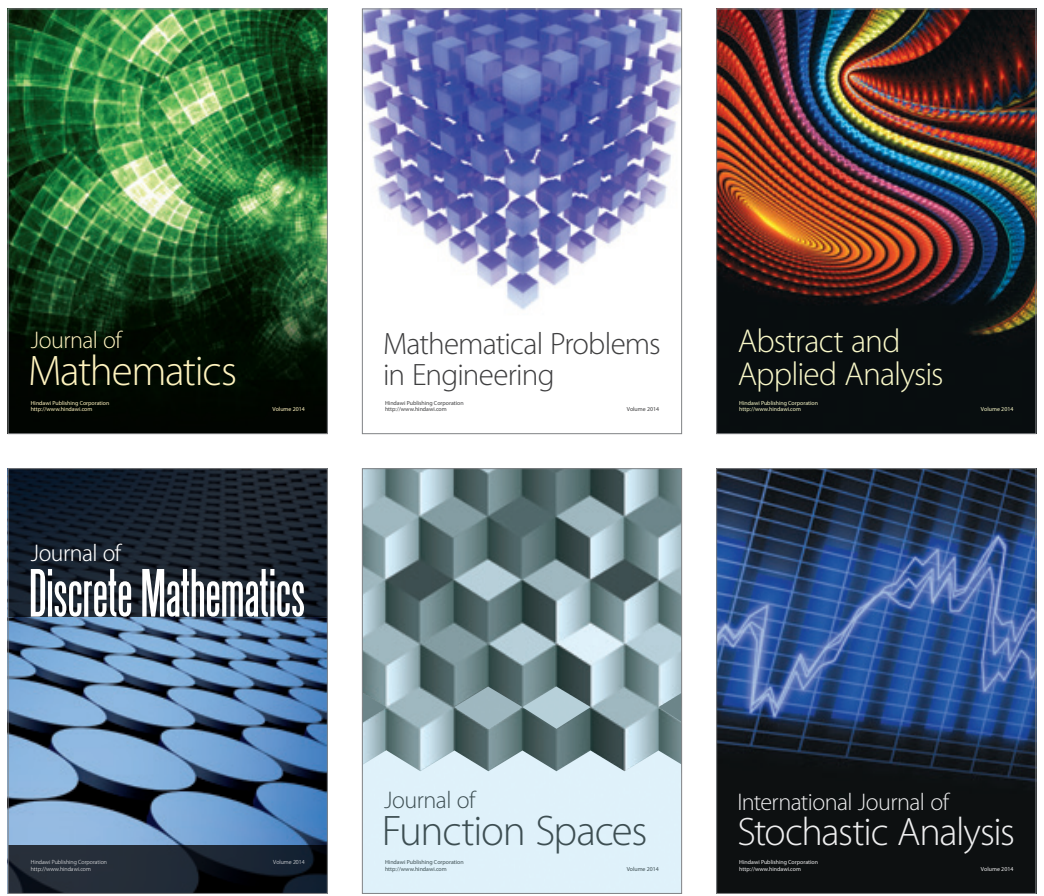

Journal of

Function Spaces

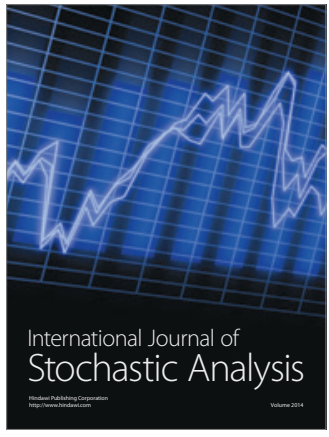

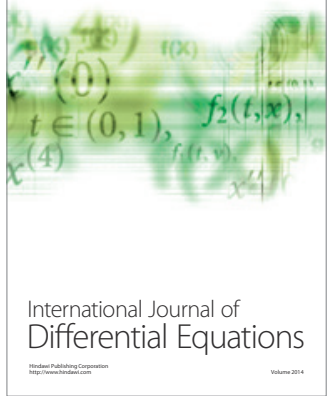
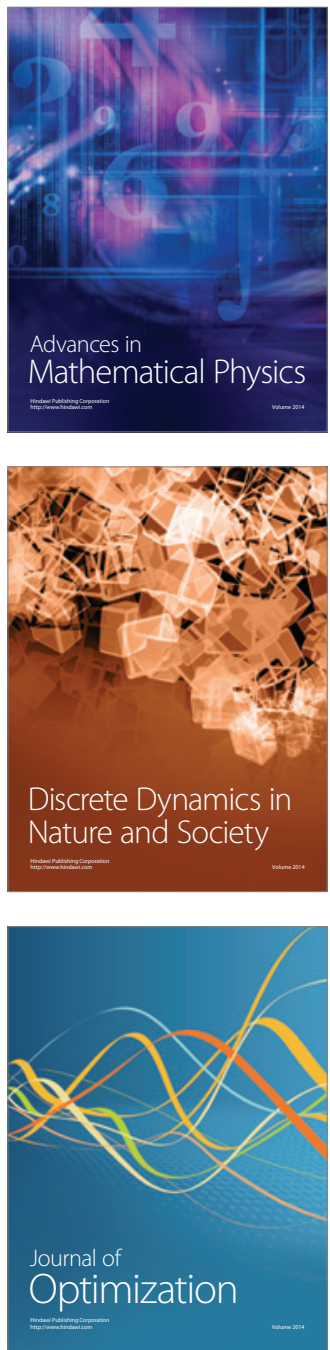